\title{
GULP: Capabilities and prospects
}

\author{
Julian D. Gale* \\ Nanochemistry Research Institute, Department of Applied Chemistry, Curtin University of Technology, P.O. Box U1987, Perth 6845, \\ Western Australia
}

Received July 9, 2004; accepted October 3, 2004

\section{Atomistic simulation / Lattice dynamics / Phonons / Electrostatics / GULP Computer program / \\ Computational crystallography}

\begin{abstract}
The current status and capabilities of the atomistic simulation code GULP are described. In particular, the differences between versions 1.3 .2 and 3.0 are detailed, as well as a concise pointer to applications in computational crystallography.
\end{abstract}

\section{Introduction}

The program GULP [1-3] is designed as a general forcefield simulation package with a strong emphasis on condensed phases. In the present description of the code the functionality that will be available in the next release, 3.0, is described. However, the core capabilities for 3-D solids and finite clusters are already included in the present release, 1.3.2.

While forcefield molecular dynamics codes are numerous, instead GULP is designed primarily for the field of lattice dynamics. In short, the program aims to provide most properties that can readily be determined based on the energy and analytic first, second and third derivatives of this quantity with respect to atomic coordinates and lattice strains. Furthermore, the code is designed to handle systems of 0-D (molecules, clusters and embedded defects), 1-D (polymers and dislocations), 2-D (slabs, surfaces and grain boundaries), and 3-D solids. In the later case, the use of crystallographic symmetry is available such that structures can be built using the space group. This also has the benefit of accelerating any structural optimisation through reducing the number of variables and only evaluating energy terms for the asymmetric unit.

\section{Capabilities}

Most calculations in GULP consist of the optimisation of a trial structure to the local energy minimum, under given conditions of pressure and temperature, followed by the calculation of the physical properties that depend on the curvature about that point. However, there has been considerable work by Woodley et al. [4] to extend this to glo-

\footnotetext{
* e-mail: julian@power.curtin.edu.au
}

bal optimization through the use of genetic algorithms. In Table 1 a concise list of the key properties that can be calculated with GULP is presented, as well as the generic capabilities.

In order to calculate the energy and its derivatives in GULP it is necessary to define the functional form of the interactions between particles and any parameters that are required. Normally the energy can be regarded as being expanded as a sum over terms that depend on increasing numbers of atomic centres. Within the code at present two-, threeand four-body interatomic potentials are allowed for, as well as certain specific many-body functional forms. The types of forcefield implemented span those typically required to simulate most classes of material, including ionic solids, semiconductors, metals and organic molecular crystals.

Aside from the interatomic potentials, a key contribution to the energy for many systems is the electrostatic energy. GULP has the capability to perform convergent electrostatic lattice sums for all dimensionalities, including the Ewald sum for 3-D [5] and the Parry sum for 2-D [6, 7]. The charges can either be fixed via the input to either formal or partial values, or can be determined as a function of structure according to electronegativity equalisation. Coulomb subtraction and scaling within molecules is also available. Furthermore, dipolar ion polarization can be included via the shell model [8], and radial compressibility via the breathing shell model [9].

A further capability that can be useful in a crystallographic context is the ability to incorporate partial occupancies for sites. This is referred to as either the meanfield or virtual crystal approximation, in which the interactions between species are weighted according to their site occupancies. Where different chemical species exist with partial occupancies on the same site, they are automatically constrained to move as a single entity within the program. Such an approach is useful for simulating crystallographic averages for disordered materials, though thermodynamic information, including excess quantities, typically requires explicit supercell orderings for an accurate description.

\section{Availability}

GULP is available to academics free of charge subject to the conditions that the program is not further distributed without permission or used for commercial research. More infor- 
Table 1. Summary of the main capabilities of GULP and the properties that can be determined directly by the program. In each case the applicable version is indicated, with all functionality of version 1.3 .2 being present in version 3.0.

\begin{tabular}{llll}
\hline Capability & Version & Property & Version \\
\hline Optimization & 1.3 .2 & Elastic constants & 1.3 .2 \\
Free energy minimization & 1.3 .2 & Bulk moduli & 1.3 .2 \\
Molecular dynamics & 1.3 .2 & Shear moduli & 3.0 \\
Monte Carlo & 3.0 & Young's moduli & 1.3 .2 \\
Defect calculations & 1.3 .2 & Piezoelectric constants & 1.3 .2 \\
Forcefield fitting & 1.3 .2 & Dielectric constants & 1.3 .2 \\
Transition state location & 1.3 .2 & Refractive indices & 1.3 .2 \\
Genetic algorithms & 1.3 .2 & Phonon frequencies & 1.3 .2 \\
Phonon free energy & 1.3 .2 & Born effective charges & 3.0 \\
& & Non-analytic frequency correction at $\Gamma$ & 3.0 \\
& & Frequency dependent properties & 3.0 \\
& & Electrostatic site potentials & 1.3 .2 \\
& & Electric field gradient tensor & 1.3 .2 \\
& & Surface and attachment energy & 3.0 \\
\hline
\end{tabular}

mation can found at the web site http:/gulp.curtin.edu.au/. While version 1.3.2, and those prior to this, were written largely in $\mathrm{f77}$, with non-standard extensions to enable some limited use of dynamic memory for arrays that are proportional to the square of the size of the problem, version 3.0 is written in 190 and has fully dynamic memory allocation. Support is provided for Unix/Linux-based operating systems, including Mac OSX. For problems that require only first derivatives, such as molecular dynamics or conjugate gradient optimization, then parallel execution can be performed using MPI. At present this is based on replicated data, with data distribution over processors either via atom number or by domain decomposition.

\section{Applications}

GULP has been widely applied in many aspects of solid state chemistry, physics, mineralogy and crystallography. Both the functionality of the code and the literature concerning its application have recently been reviewed [3]. Hence, we will present just a brief illustration here.

While there are many programs based on different Hamiltonians that make it possible to optimize the structure of a material, the capability of GULP that is more rarely found is the ability to minimize under symmetry constraints and to rapidly analyze the phonon behaviour of the final structure. It is often crucial to determine the phonon modes at the Brillouin zone centre, especially following a symmetry constrained optimization, in order to verify that the system is stable within the given space group. In order for a system to be at an energy minimum it is necessary that the first three phonon modes (i.e. the acoustic branch) be equal to zero, as a consequence of transitional invariance. Furthermore, the remaining vibrational frequencies must all be real, instead of imaginary. When this is not the case, this indicates that the imposed symmetry is too high and that the space group must be changed. This information can only be obtained from the phonon spectrum and not from the Hessian matrix, which will show no instabilities under the space group constraint.
During studies of silica and aluminophosphate polymorphs $[10,11]$, many of which are microporous phases, there have been a number of instances of theoretical prediction of symmetry lowering based on lattice dynamical information. One of the best known examples is that of the material silicalite, which exhibits an orthorhombic structure at elevated temperatures, such as those employed when it is used as a heterogeneous catalyst. However, energy minimization followed by a phonon calculation indicates that at lower temperatures, as implied by the neglect of thermal effects in the simulation, the system should distort to eleviate the imaginary mode that is found when studying the orthorhombic unit cell. Indeed, it is now well-known that silicalite undergoes a monoclinic-orthorhombic transition with temperature. However, the calculations demonstrate that the lower symmetry is an intrinsic property of the framework structure, rather than being a consequence of the presence of impurities in the material. Although we should point out that the original calculations on silicalite [12] were not actually performed with GULP, the result is reproduced with the present code. More recently, the ability to determine analytical derivatives of vibrational free energy within GULP [13] has been used to examine the temperature dependent variation of the monoclinic distortion in silicalite. Although the quasiharmonic approximation breaks down before phase transition is reached, the results of Grau-Crespo et al. [14] indicate that the monoclinic angle does indeed tend towards a right angle as the temperature rises. It should also be noted that the use of a shell-model is crucial for the proper description of such distortions since they are driven by polarization.

The existance of imaginary modes in the phonon spectrum is not confined to the zone centre. Occasionally, the maximum in the modulus of the imaginary mode may be at some other special point in the Brillouin zone. Here the inverse of the fractional coordinates in reciprocal space indicates the dimension of the supercell that the system wishes to adopt. This situation occured in a recent study of the (10-14) surface of the mineral calcite $\left(\mathrm{CaCO}_{3}\right)$ [15]. Examination of the phonons for the relaxed surface showed the presence of an imaginary mode at $(0.5,0.0)$ in the Brillouin zone. After the creation of a $(2 \times 1)$ super- 
cell, followed by perturbation along the direction of the imaginary mode eigenvector and re-optimization, the final surface structure was determined. Alternate carbonate groups were found to be rotated differently, leading to a superlattice that is consist with observations from experimental LEED work [16].

\section{Future directions}

While the program encompasses a wide variety of forcefields and simulation types, there are still many developments of GULP in progress. In order to broaden the range of applicability of the code, and to improve the accuracy, new model Hamiltonians are always being added. Presently, the many different forms of bond order potentials are being introduced, with those due to Tersoff [17] and Brenner et al. [18] already implemented. These are particularly useful for many covalent systems of current interest, such as silicon and carbon nanotubes. The trend towards including models that are increasingly influenced by quantum mechanical or many-body effects will also continue. Examples, of some of the development work includes the incorporation of ligand field effects into the description of transition metal systems [19], especially those that exhibit a Jahn-Teller distortion, and use of periodic MNDO-based Hamiltonians to achieve a self-consistent dipolar and quadrupolar description of atomic polarization in the solid state [20].

Acknowledgments. We would like to thank the Government of Western Australia for its support through the Premier's Research Fellowship programme, as well as funding from Curtin University of Technology. The contribution of the ARC to recent developments through the provision of an IREX grant is also appreciated.

\section{References}

[1] Gale, J.: GULP: A computer program for the symmetry-adapted simulation of solids. J. Chem. Soc., Faraday Trans. 1 (1997) $629-637$.
[2] Gale, J.: Empirical potential derivation for ionic materials. Phil. Mag. B73 (1996) 3-19.

[3] Gale, J.; Rohl, A.: The general utility lattice program. Mol. Simul. 29 (2003) 291-341.

[4] Woodley, S.; Battle, P.; Gale, J.; Catlow, C.: The prediction of inorganic crystal structures using a genetic algorithm and energy minimisation. Phys. Chem. Chem. Phys. 1 (1999) 2535-2542.

[5] Ewald, P.: Die Berechnung optischer und elektrostatischer Gitterpotentiale. Ann. Phys. 64 (1921) 253-287.

[6] Parry, D.: The electrostatic potential in the surface region of an ionic crystal. Surf. Sci. 49 (1975) 433-440.

[7] Parry, D.: Errata: The electrostatic potential in the surface region of an ionic crystal. Surf. Sci. 54 (1976) 195-195.

[8] Dick, B.; Overhauser, A.: Theory of the dielectric constants of alkali halide crystals. Phys. Rev. 112 (1958) 90-103.

[9] Schroeder, U.: A new model for lattice dynamics (breathing shell model). Solid State Commun. 4 (1966) 347-349.

[10] Henson, N.; Cheetham, A.; Gale, J.: Computational studies of aluminium phosphate polymorphs. Chem. Mater. 8 (1996) 664670.

[11] Henson, N.; Cheetham, A.; Gale, J.: Theoretical calculations on silica frameworks and their correlation with experiment. Chem. Mater. 6 (1994) 1647-1650.

[12] Bell, R.; Jackson, R.; Catlow, C.: Computer simulation of the monoclinic distortion in silicalite. JCS Chem. Comm. (1990) $782-783$

[13] Gale, J.: Analytical free energy minimization of silica polymorphs. J. Phys. Chem. B102 (1998) 5423-5431.

[14] Grau-Crespo, R.; Acuay, E.; Ruiz-Salvador, A.: A free energy minimization study of the monoclinic-orthorhombic transition in mfi zeolite. JCS Chem. Commun. (2002) 2544-2545.

[15] Rohl, A.; Wright, K.; Gale, J.: Evidence from surface phonons for the $(2 \times 1)$ reconstruction of the (10-14) surface of calcite from computer simulation. Am. Miner. 88 (2003) 921-925.

[16] Stipp, S.: Toward a conceptual model of the calcite surface: Hydration, hydrolysis, and surface potential. Geochimica Cosmochimica Acta 63 (1999) 3121-3131.

[17] Tersoff, J.: New empirical-model for the structural-properties of silicon. Phys. Rev. Lett. 56 (1986) 632-635.

[18] Brenner, D.; Shenderova, O.; Harrison, J.; Stuart, S.; Ni, B.; Sinnott, S.: A second-generation reactive empirical bond order (rebo) potential energy expression for hydrocarbons. J. Phys.: Condens. Matter 14 (2002) 783-802.

[19] Woodley, S.; Battle, P.; Catlow, C.; Gale, J.: Development of a new interatomic potential for the modeling of ligand field effects. J. Phys. Chem. B105 (2001) 6824-6830.

[20] Gale, J.: Semi-empirical methods as a tool in solid state chemistry. Faraday Discuss. 106 (1997) 219-232. 